\title{
Théories et systèmes : le cas de la sociologie
}

\section{Claude Grignon}

\section{OpenEdition \\ Journals}

Édition électronique

URL : http://journals.openedition.org/ress/126

DOI : $10.4000 /$ ress. 126

ISSN : 1663-4446

Éditeur

Librairie Droz

Édition imprimée

Date de publication : 1 septembre 2008

Pagination : 7-10

ISBN : 978-2-600-01242-3

ISSN : 0048-8046

Référence électronique

Claude Grignon, "Théories et systèmes : le cas de la sociologie », Revue européenne des sciences sociales [En ligne], XLVI-142 | 2008, mis en ligne le 01 septembre 2011, consulté le 19 avril 2019. URL http://journals.openedition.org/ress/126 ; DOI : 10.4000/ress.126 


\section{INTRODUCTION}

\section{THÉORIES ET SYSTÈMES : LE CAS DE LA SOCIOLOGIE}

Le cas de la théorie de l'évolution montre que les sciences historiques, narratives et observationnelles peuvent aboutir à des théories générales; mais on ne voit guère d'équivalent de cette réussite dans les sciences de l'homme*. Celles-ci n'ont pas cessé en revanche d'accueillir ou de développer des systèmes de pensée qui prétendent fournir une explication ultime, globale et définitive de la «société» ou de «l'histoire». On trouve chez Claude Bernard une formulation claire et toujours actuelle de l'opposition entre théorie scientifique et système: «La médecine expérimentale ne sera ni vitaliste, ni animiste, ni organiciste, ni solidiste, ni humorale... Elle n'a que faire de s'embarrasser de systèmes, qui, ni les uns ni les autres, ne sauraient jamais exprimer la vérité (...) Le temps des doctrines et des systèmes personnels est passé, et peu à peu ils sont remplacés par des théories représentant l'état actuel de la science, et donnant à ce point de vue le résultat des efforts de tous » (Bernard 1966 [1865]: 303)1.

Ce qu'on entend par théorie se précisera par la suite, notamment par la comparaison entre théories et systèmes (chapitre 1), et par l'étude des manières de théoriser propres aux différentes sciences (chapitre 3). Disons pour commencer qu'une théorie scientifique est un énoncé capable de rassembler des phénomènes considérés jusque là comme hétérogènes, et de définir la relation qui les unit:

L'hypothèse est permise dans les recherches scientifiques, et si elle explique un ensemble de faits considérables et indépendants, elle s'élève au rang d'une théorie bien fondée (...) Le principe de la sélection naturelle peut être regardé comme une pure hypothèse; mais ce que nous savons de positif sur la variabilité des êtres organisés à l'état de nature, les

* Cet ouvrage est issu des réflexions que le séminaire d'épistémologie comparée «Sciences de l'Homme et Sciences de la Nature » m'a amené à développer. Mes remerciements vont donc d'abord, par ordre chronologique, à mes collègues des autres disciplines, Roland Omnès, Maurice Aymard et Claude Kordon, qui m'ont aidé dès le début à l'organiser et qui n'ont cessé de le soutenir. Les lectures attentives, aux étapes successives du manuscrit, de Raymonde Moulin, Jean-Michel Chevet, Michel Grignon, et de ma femme et collègue, Christiane, à qui ce volume est dédié, ont beaucoup contribué à la maturation et à la clarification de mes idées. Je dois aussi beaucoup aux conseils de Bertrand SaintSernin, qui m'a incité à mettre la dernière main à mon texte et à le publier. Je suis profondément reconnaissant à Anne Fagot-Largeault pour ses critiques encourageantes et justes, qui m'ont conduit à approfondir et à préciser des points essentiels, comme les rapports entre les différentes espèces de théories.

1 C. Bernard, Introduction à la médecine expérimentale, Paris, Garnier Flammarion, 1966 [1865], pp. 303 sq. Sur la prolifération des systèmes en biologie, voir E. Mayr, Histoire de la biologie, diversité, évolution et hérédité, Paris, Fayard, 1989 (traduction par M. Blanc de The Growth of Biological Thought. Diversity, Evolution and Inheritance, The Bellknap Press of Harvard University Press). 


\begin{abstract}
renseignements certains que nous possédons sur la lutte pour l'existence et la conservation presque inévitable des variations favorables qui en est la conséquence, enfin, la formation analogue des races domestiques, donnent un certain degré de probabilité à cette hypothèse. Or, on peut la mettre à l'épreuve (et ceci me paraît la seule manière équitable et légitime de considérer l'ensemble de la question) en cherchant si elle explique certains groupes de faits indépendants les uns des autres, tels que la succession géologique des êtres organisés, leur distribution dans les temps passés et actuels, leurs affinités mutuelles et leurs homologies. Si le principe de la sélection naturelle explique ces groupes de faits importants et d'autres encore, il doit être pris en considération (Darwin $1879)^{2}$.
\end{abstract}

Je me propose de montrer qu'une théorie scientifique ne s'énonce et ne se conçoit pas nécessairement en mathématiques, mais, aussi bien, en langue dite naturelle. Contrairement à ce que fait croire la hiérarchie des sciences héritée du positivisme viennois, les théories-récits des sciences historiques ne sont ni moins scientifiques ni moins théoriques que les théories-modèles des sciences nomothétiques. Ces deux espèces de sciences théorisent sans doute en suivant des démarches opposées, bottom up, par induction et par généralisation progressive pour les premières, top down, par déduction à partir d'une axiomatique pour les secondes; mais elles ont en commun de transformer leurs spéculations en hypothèses, et de soumettre celles-ci au démenti, à la réfutation de la réalité. Comme le note Darwin, les théories narratives s'efforcent elles aussi de dégager un principe explicatif. Il ne suffit pas, en effet, de mettre en relation des faits apparemment hétérogènes, encore faut-il montrer que ces faits sont homogènes au sens étymologique du terme, c'est-à-dire qu'ils ont une origine commune $(\gamma \in \nu \circ \zeta=$ naissance, origine). Reste que les théories-récits et les théories-modèles mettent en œuvre des conceptions de l'explication et de la causalité différentes; alors que les théories des sciences nomothétiques réduisent l'explication à la prédiction et montrent que les phénomènes qu'elles rassemblent obéissent tous aux lois sur lesquelles elles reposent, les théories des sciences historiques réintroduisent l'idée de temps irréversible, optent pour une conception chronologique et probabiliste de la causalité, et énoncent en langage naturel le principe auxquelles elles ramènent la diversité des enchaînements et des processus dont les observations qu'elles réunissent sont la conséquence et le résultat.

Les systèmes de pensée se présentent et sont reçus comme des théories scientifiques, dont ils tiennent lieu et prennent la place. Il faut distinguer entre ces pseudo-théories et:

- les théories proprement philosophiques, qui, même si elles prennent les sciences comme objet de réflexion, ne prétendent pas se substituer à celles-ci et ne se donnent pas comme des théories scientifiques (c'est le cas, par exemple, de la pensée de Cournot);

- les théories fausses, erronées ou périmées, mais dont on a pu montrer qu'elles étaient fausses, et qui sont donc de vraies théories scientifiques. Alors que les fausses théories reposent sur des principes d'interprétation généraux (comme

2 C. Darwin, De la variation des animaux et des plantes à l'état domestique, Paris, C. Reinwald et Cie, 1879, traduit de la deuxième édition anglaise par E. Barbier. 
«l'inconscient», «la lutte des classes» ou «l'habitus»), les théories fausses reposent sur des entités explicatives spécifiques, qu'on est conduit à imaginer pour essayer de résoudre les problèmes qui se posent, les énigmes qui subsistent dans un état donné de la science, et que l'expérimentation ou l'observation conduit à abandonner (comme le phlogistique, ou l'éther, milieu supposé de propagation des ondes lumineuses, réfuté par l'expérience de MichelsonMorley sur le mouvement relatif de la Terre) $)^{3}$.

Cette méprise oppose un barrage invisible au progrès des sciences de l'homme. En les condamnant à se contenter de pseudo-théories, en supprimant jusqu'au besoin d'en produire de véritables, elle en fait non des sciences imparfaites, momentanément retardées par des théories fausses, mais des pseudosciences. Elle les conforte dans le sentiment et dans la prétention d'être des sciences, tout en les rejetant du côté des innombrables systèmes de pensée dont l'homme a été et continue d'être l'objet, et les maintient ainsi dans un statut ambivalent mais incertain qui menace sans doute à terme leur existence même, en les empêchant de se distinguer de la philosophie, de l'essayisme, de la littérature, des «sagesses » et des arts de vivre.

La science de l'homme, c'est-à-dire l'anthropologie au sens propre du terme (et non au sens abusivement confisqué par les anthropologues) n'est pas moins divisée que la biologie; de même que celle-ci est partagée entre la biologie des organismes et la biologie des populations, l'anthropologie, lato sed proprio sensu, se partage entre les sciences de l'homme collectif, qui s'intéressent à des ensembles (populations, groupes, institutions) irréductibles aux individus qui les composent, et les sciences de l'homme individuel, comme la psychologie ou les sciences de la cognition. Il existe sans doute des disciplines intermédiaires ou plutôt ambivalentes, comme la psychologie sociale, voire l'économie; mais elles oscillent elles aussi entre ces deux pôles. La sociologie même a pu se diviser entre les écoles opposées de l'individualisme méthodologique et de ce que les indivi-

3 «On ne sort pas du champ expérimental, ni par conséquent du domaine des théories logicoexpérimentales (I a), en faisant usage d'hypothèses, employées uniquement comme moyen de déduction, et toujours subordonnées aux vérifications de l'expérience. On en sortirait, si ces hypothèses jouaient le rôle de raison démonstrative, indépendamment des vérifications expérimentales (...) Cela n'a pas été compris de ceux qui, comme A. Comte, voulaient exclure de la science l'hypothèse de l'éther lumineux. Cette hypothèse et d'autres semblables ne doivent pas être jugées d'une façon intrinsèque, mais extrinsèque; c'est-à-dire en observant si et jusqu'où leurs conséquences concordent avec les faits ». V. Pareto, Traité de sociologie générale, Paris - Genève, Droz, 1968 [1917], Ch. I, § 59.

La réfutation de ces entités explicatives provisoires fait progresser la théorie; ainsi l'expérience de Michelson-Morley a constitué l'un des points de départ de la théorie de la relativité restreinte. Elle est plus difficile dans le cas des sciences historiques fondées sur l'observation, quand celles-ci sont amenées à postuler un phénomène dont l'existence de fait reste à constater (comme les «chaînons manquants» de la théorie de l'évolution, ou le schwa indo germanique imaginé pour expliquer les différences entre les voyelles entre langues d'une même famille). Alors que la non observation de spécimens correspondant à l'hypothèse peut se prolonger indéfiniment sans l'infirmer, la découverte d'un seul la confirme, et c'est à la recherche de ceux-ci que l'on part. La réfutation ne peut intervenir qu'indirectement, par la non découverte de spécimens dans des contextes où l'on aurait dû en trouver, d'après les prédictions de la théorie, ou par la découverte de spécimens confirmant des hypothèses et des théories concurrentes. 
dualistes appellent le holisme (un euphémisme pour totalitarisme). Mais on voit mal comment les sociologues peuvent revenir sur l'irréductibilité des faits sociaux aux faits psychiques individuels sans mettre en cause le principe fondateur de leur discipline (Durkheim, 1960 [1894]) ${ }^{4}$. Partant de la discipline que j'ai pratiquée, j'examinerai ici le cas des sciences de l'homme collectif. Parmi celles-ci je retiendrai surtout celles qui sont les plus exposées à confondre théorie et système parce qu'elles ont la vocation la plus générale, c'est-à-dire la sociologie, l'histoire et l'ethnologie, plutôt que les sciences spécialisées, comme la démographie ou la linguistique (même si, là encore, on peut trouver des cas intermédiaires, comme la sociolinguistique).

Si cette confusion passe inaperçue, si elle est aussi fréquente et aussi durable, c'est sans doute parce qu'un système de pensée ressemble en effet à s'y méprendre à une théorie scientifique. Cette ressemblance n'est pas seulement apparente, superficielle et voulue; elle ne se réduit pas à des emprunts plus ou moins maladroits, à un habillage rhétorique, à un travestissement scientifique, comme dans le cas des impostures intellectuelles dénoncées par Sokal et Bricmont ${ }^{5}$. Si les systèmes de pensée, dans nos disciplines, peuvent passer aussi aisément pour des théories scientifiques, c'est sans doute parce que les rapports entre la pensée spéculative et la pensée scientifique y sont particulièrement étroits, intimes et obscurs. Pour dissiper cette confusion, il faut donc commencer par aller le plus loin possible dans la reconnaissance et dans l'inventaire de leurs similitudes réelles, non pour montrer, comme y invite une atmosphère intellectuelle saturée de relativisme, que les théories scientifiques ne sont que des systèmes de pensée, mais, tout au contraire, pour repérer, par élimination, leurs véritables différences, et déterminer ainsi les conditions sous lesquelles les tentatives de théorisation, dans les sciences de l'homme, peuvent se distinguer des systèmes de pensée.

4 «Voilà donc un ordre de faits qui présentent des caractères très spéciaux: ils consistent en des manières d'agir, de penser et de sentir, extérieures à l'individu, et qui sont douées d'un pouvoir de coercition en vertu duquel ils s'imposent à lui. Par suite, ils ne sauraient se confondre avec les phénomènes organiques, puisqu'ils consistent en représentations et en actions; ni avec les phénomènes psychiques, lesquels n'ont d'existence que dans la conscience individuelle et par elle. Ils constituent donc une espèce nouvelle et c'est à eux que doit être donnée et réservée la qualification de sociaux». E. Durkheim, Les règles de la méthode sociologique, Paris, PUF, 1960 [1894], pp. 5, 6.

5 A. Sokal, J. Bricmont, Impostures intellectuelles, Paris, Odile Jacob, 1997. 